\title{
A REDUCED MODEL FOR A PNEUMATIC TRANSMISSION LINE
}

\author{
Toshiharu Kagawa, Ato Kitagawa* \\ Kazushi Sanada*, Toshio Takenaka** \\ ${ }^{*}$ Dept. of Control Engineering, Faculty of Engineering \\ Tokyo Institute of Technology \\ Tokyo, Japan \\ ${ }^{* *}$ Dept. of Mechanical Engineering, Faculty of Engineering \\ Musashi Institute of Technology \\ Tokyo, Japan
}

\section{ABSTRACT}

A pneumatic transmission line is widely used for measurement and control systems. Therefore it is necessary to calculate dynamic responses of the line. The Brown's model of the pneumatic transmission line gives sufficiently accurate responses, but it needs troublesome calculations including Bessel functions. In this study, a new reduced model is proposed, which is presented by first or second order system and time delay system. So calculatations using the reduced model can be very easily carried out. In experiments, tests of step responses and frequency responses were conducted. The reduced model showed a good agreement with the exact solution and experimental results. The new reduced model is very useful for designing a real control system with pneumatic lines.

\section{KEYWORDS}

Pneumatic Transmission Line, Process Control, Reduced Mode1, Model Matching

\section{NOMENCLATURE}

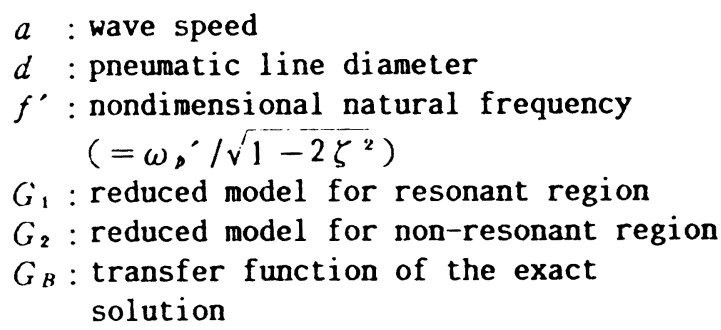

$J_{0}, J_{1}$ : Bessel functions of the first kind

$l$ : pipe length

$p_{1}$ : inlet pressure

$p_{2}$ : outlet pressure

$p^{\prime}=\Delta p_{2} / \Delta p_{1}$

$p^{\prime \prime}=\Delta p_{2} / S V$

$\mathrm{P}_{\mathrm{r}}$ : Prandt1 number

$r=d / 2$

$R_{N}$ : parameter for pneumatic transmission line characteristics

$\left(=a r^{2} /\left(\begin{array}{ll}\nu & l\end{array}\right)\right)$

$s$ : Laplace variable

$s^{\prime}$ : nondimensional Laplace variable

$S V$ : set value of input pressure

$T_{1}^{\prime}$ : nondimensional time constant

$$
\left(=T_{1} / T_{l}\right)
$$

$T_{d}{ }^{\prime}$ : nondimensional delayed time $\left(=T_{d} / T_{l}\right)$

$T_{l}=l / a$

$t:$ time

$t^{\prime}=t / T_{l}$

$\kappa$ : ratio of specific heats

$\nu$ : kinematic viscosity

$\zeta$ : damping coefficient

$\omega$ : angular frequency

$\omega^{\prime}=T_{\iota} \omega$

$\omega_{p}^{\prime}$ : nondimensional resonant angular frequency

$\left(=T_{l} \omega_{p}\right)$

\section{INTRODUCTION}

Pneumatic transmission lines are widely used for measurement and control systems. So signal transmission characteristics of the line have been investigated by various authors $(1) \sim(3)$. When the amplitude of a pressure signal is small enough, the transmission characteristics are precisely 
$4 \overline{50}$

presented by the Brown's theoretical model (4). But since the solution of the Brown model contains Bessel functions, it is not easy to obtain frequency or transient responses. From a practical point of view, approximating method of signal transmission characteristics of pneumatic transmission line by a reduced model is a quite important research subject. By Nishihara(5), an approximating technique of signal transmission characteristics by use of a time delay system and a first order lag system was presented. Bacci also proposed a reduced model with two poles and two zeros(6). These models are effective only when a pneumatic transmission line has nonresonant characteristics ( that is, the line diameter is small and the line is long). But the applicable limit of those models have not been specifically pointed out. By Anderson, a reduced model including resonant region characteristics was proposed(7). In Anderson's model, a polytropic compression of air in a line was assumed. For this assumption, evaluating error of polytropic exponent may have a considerably unfavorable influence on calculating accuracy of signal transmission characteristics. Besides these models, an approximating technique of the ratio of Bessel functions by functions which include square root functions was proposed(8). And an approximating method of transmission characteristics by products of finit terms of rational functions was proposed(9). But there have been few reports in which a reduced model was compared with the exact solution with the object of designing a real control system containing pneumatic lines.

In this paper, from considerations of a nondimensional parameter which represents dynamic responses of a pneumatic transmission line, a new reduced model which contains the effect of unsteady velocity and temperature distribution on a pipe cross section has been proposed. And the new reduced model is compared with the exact solution in time and frequency regions. The new reduced model presented in this investigation needs only two or three parameters. But by the new model, signal transmission characteristics of pneumatic transmission lines are able to be calculated accurately enough for practical use.

In the following discussion, the theoretical result in Laplace domain by Brown model (4) and the numerical result in time domain by the characteristics method (10) are termed as the exact solution.

\section{REDUCED MODEL}

A pneumatic transmission line treated in this paper is a line termined by block end as shown in Fig.1. The line is a systen whose input signal is inlet pressure $p_{1}$ and whose output signal is pressure $f_{2}$ at the block end. In this pneumatic line system, it is assumed that temperature is constant and velocity is zero on the pipe wall. And it is also assumed that the pipe diameter does not change and that leakage of air is zero. By Brown, the transfer function of the system $G_{B}$ can be written as

$$
G_{B}=\frac{P_{2}(s)}{P_{1}(s)}=\frac{1}{\cosh I l}
$$

$I^{V}$ is the propagation function of a pneumatic transmission line;

$$
\Gamma=\left\{\frac{1+2(\kappa-1) J_{1}(X) / X J_{0}(X)}{1-2 J_{1}(Y) / Y J_{0}(Y)}\right\}^{1 / 2} \frac{s}{a},
$$

where

$$
\begin{aligned}
& X=j r \sqrt{\mathrm{Pr} s / \nu}, \\
& Y=j r \sqrt{s / \nu} .
\end{aligned}
$$

Signal transmission characteristics of a pneumatic transmission line are able to be represented almost accurately by Eq. (1). But the transfer function Eq. (1) contains Bessel functions, so the Brown model is not suitable for rough estimates of line characteristics. It is difficult to directly utilize the Brown model for

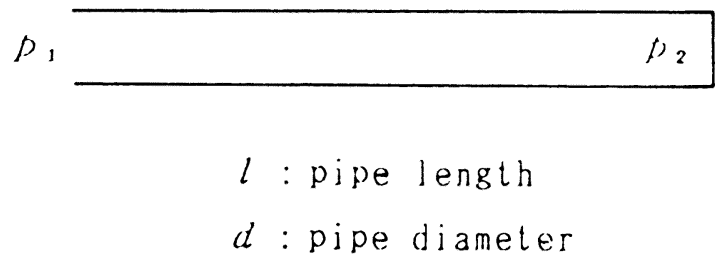

Fig. 1 Pneumatic transmission line terminated by block end

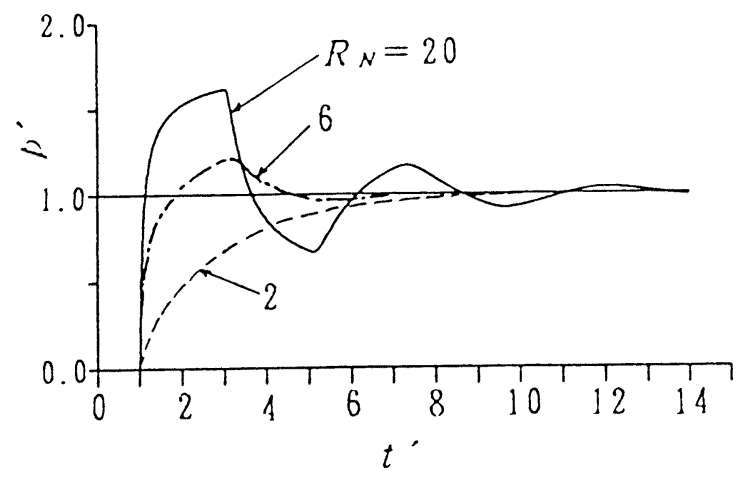

Fig. 2 Step responses of a pneumatic transmission line by dimensionless parameter 
calculating dynamic characteristics of a control system with pneumatic lines. Therefore it is necessary for a reduced model that only a few parameters are needed and order of the model is low. In Fig.2, step responses of a pneumatic line shown in Fig. 1 are presented for several nondimensional parameters $R_{N} \quad$ (10). Transient responses indicated by the Brown model were calculated by use of the accurate and high speed computation method for the characteristics method (10). $K_{N}$ is a parameter which represents the ratio of the boundary layer developing time to the wave traveling time.

Because of energy consumption caused by viscosity and heat dissipation, transient pressure wave is damped. The degree of pressure wave damp varies with $R_{N}$. Without viscosity and heat dissipation, pressure wave can travel undamped. Therefore measurement results of transient pressure at block end become rectangular wave with a period of $4 T /$. For obtaining nondimensional equations, there is one way using the velocity boundary layer developing time as a standard time. In the case of designing real control systems, line length is determined by specifications of a plant to be controlled. Then dynamic responses of lines are calculated for various line diameters. Therefore in this investigation, the pressure wave traveling time was used as a standard time. Nondimensional Laplace variable $s^{\prime}$ is expressed as

$$
s^{\cdot}=T_{l} s
$$

By use of Eq. (3) and parameter $R_{N}$, Eq. (1) and Eq. (2) are rearranged as

$$
G_{B}\left(s^{\prime}\right)=\frac{P_{2}\left(s^{\prime}\right)}{P_{1}\left(s^{\prime}\right)}=\frac{1}{\cosh \Lambda},
$$

where

$$
\left\{\begin{array}{l}
\Lambda=\left\{\frac{1+2(\kappa-1) J_{1}(X) / X J_{0}(X)}{1-2 J_{1}(Y) / J_{0}(Y)}\right\}^{1 / 2} s^{\prime} \\
X=j \sqrt{\operatorname{Pr} R_{N} s^{\prime}}, \quad Y=j \sqrt{R_{N} s^{\prime}} .
\end{array}\right.
$$

Dynamic responses of pneumatic lines are classified into two regions. The bound of these two regions is presented by $R_{N}=$ 3.64. A pneumatic line with small $R_{N}$ corresponds to the long and narrow line. Dynamic responses of such a long and narrow line are overdamped $\left(R_{N}=2\right.$ in Fig. 2$)$. On the contrary, when $R_{N}$ is large $\left(R_{N}=6\right.$ and 20 in Fig.2 ), dynamic responses are underdamped. In Fig.3, frequency responses of a pneumatic line system shown in Fig. 1 are presented for three cases of $R_{N}$.
Similar to the case of step responses, when $R_{N}$ equals 20 or 6 , frequency responses show resonant characteristics. When $R_{N}$ equals 2 , frequency responses show non-resonant characteristics. In this investigation, for these two regions, a resonant model $G_{1}\left(s^{\circ}\right)$ and a non-resonant model $G_{2}\left(s^{\prime}\right)$ were proposed.

A reduced model $G_{1}\left(s^{\prime}\right)$ for the resonant region is determined by nondimensional natural frequency $f^{\prime}$, damping coefficient $\zeta$ and nondimensional delayed time $T_{d}^{\prime}$. This model is given by

$$
G_{1}\left(s^{\prime}\right)=\frac{f^{\prime 2} \exp \left(-T_{d^{\prime}} s^{\prime}\right)}{s^{\prime 2}+2 \zeta f^{\prime} s^{\prime}+f^{\prime 2}}
$$

for

$$
R_{N}>3: 64
$$

Three parameters of the resonant region model $G_{1}\left(s^{\prime}\right)$ have been so determined that the gain and the phase lag of the model equal those of the exact solution at the first resonant frequency. In Fig.4, relations between three parameters and $R_{N}$ are presented.

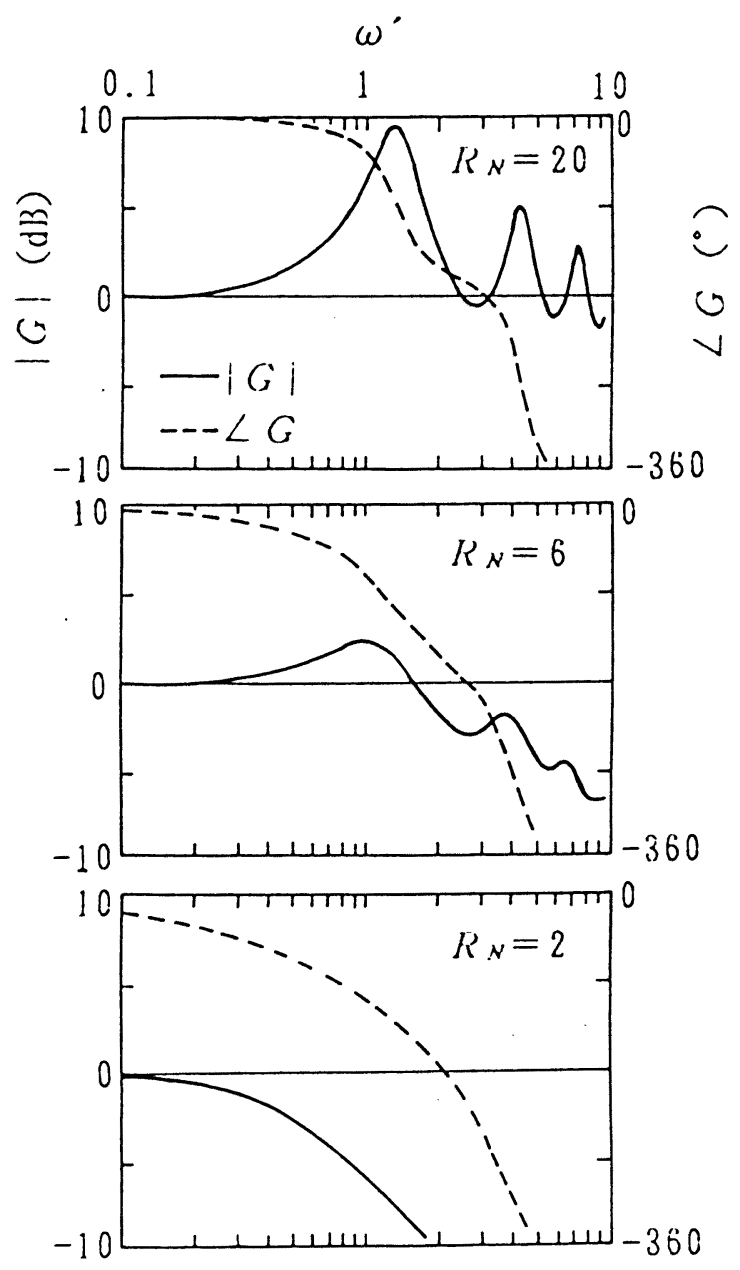

Fig. 3 Frequency responses of a pneumatic transmission line 
A reduced model $G_{2}\left(s^{\prime}\right)$ for the nonresonant region is determined by nondimensional time constant $T_{1}{ }^{\prime}$ and nondimensional delayed time $T_{d}^{\prime}$. The model is given by

$$
G_{2}\left(s^{\prime}\right)=\frac{\exp \left(-T_{d^{\prime}} s^{\prime}\right)}{T_{1}^{\prime} s^{\prime}+1}
$$

for

$$
R_{N} \leqq 3.64
$$

Two parameters of the non-resonant region model $G_{2}\left(s^{\circ}\right)$ have been so determined that the phase lag of the reduced model equals that of the exact solution at the break frequency. In Fig.5, relations between two parameters and $R_{N}$ are presented.

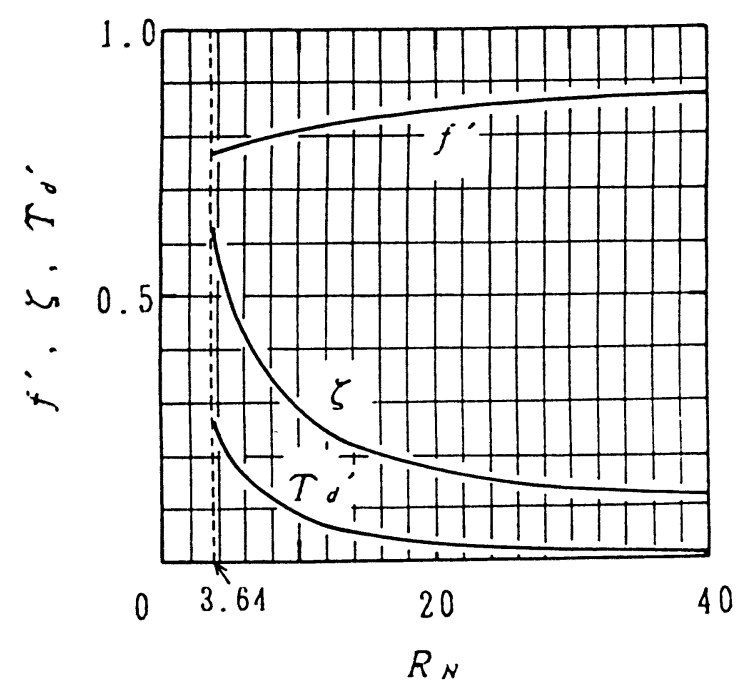

Fig. 4 Nondimensional natural frequency, damping ratio and time $1 \mathrm{ag}$

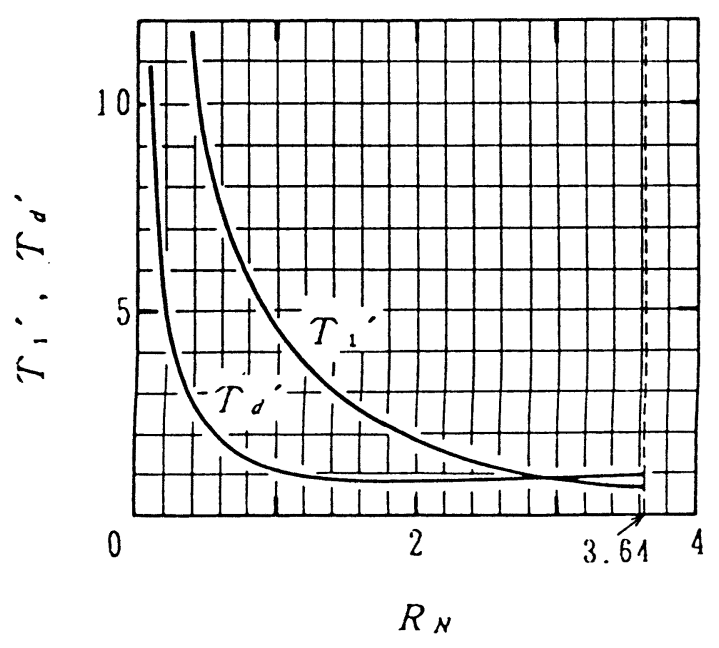

Fig. 5 Nondimensional time constant and time lag
COMPARISON WITH THE EXACT SOLUTION

In this chapter, reduced models $G_{1}\left(s^{\circ}\right)$ and $G_{2}\left(s^{\circ}\right)$, which have been determined cosidering frequency responses, are investigated and compared with the exact solution in time region. The exact solution of step responses by the Brown model was obtained using the high speed and accurate computing method (10) for a pneumatic transmission line. In Fig.6, computed results of step responses for $K_{N}=20,6$ and 2 are presented. Step responses for $K_{N}=20$ and 6 showed resonant characteristics. Especially in the step response for $R_{N}=20$, reflection and propagation of pressure, wave were clearly distinguished. Of course, by the reduced model $G_{1}\left(s^{\circ}\right)$, details of pressure wave can not be reproduced, but the outline of transient pressure wave can be obtained. Computed results for $R_{N}=2$ showed non-resonant characteristics. Responses obtained by a reduced model $G_{2}\left(s^{\prime}\right)$ well agreed with the exact solution except a rising part of the responses.

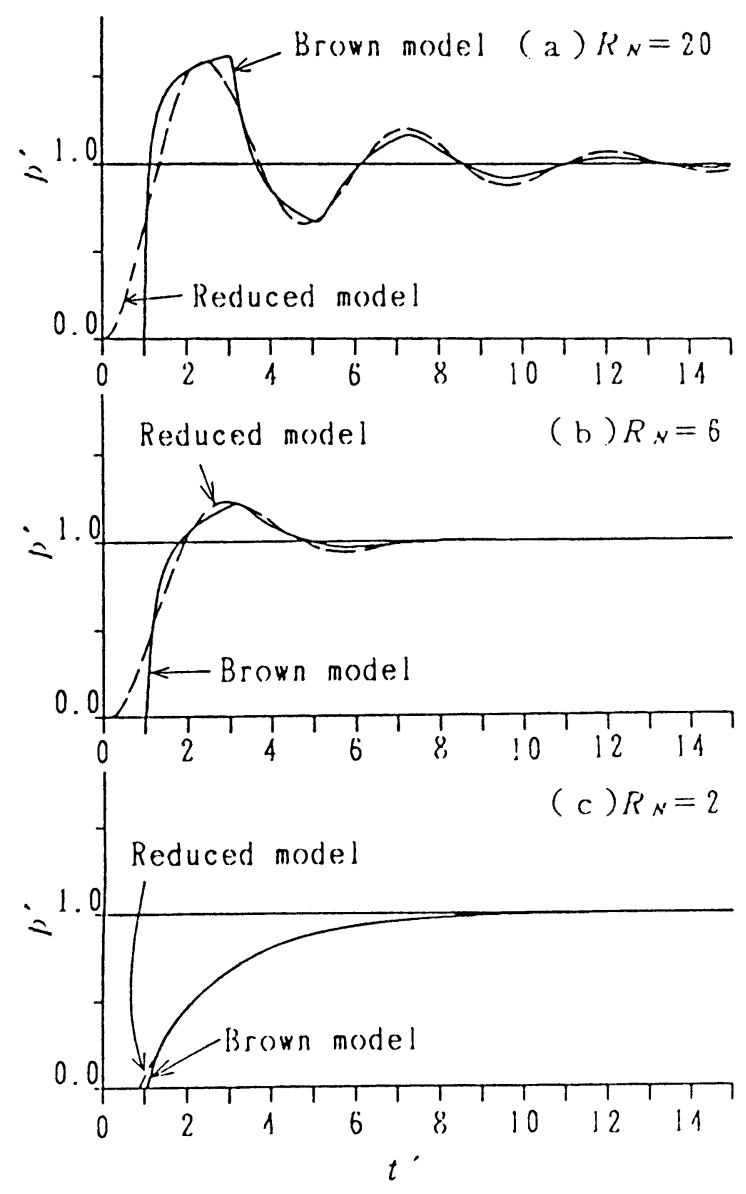

Fig.6 Step responses of a pneumatic transmission line 


\section{CLOSED LOOP SYSTEM}

When the signal transmission characteristics of pneumatic lines can not be omitted, an investigation of dynamic characteristics of the system with pneumatic lines may become a very important subject. In the control system which contains pneumatic lines as its elements, it is necessary to investigate total influences of pneumatic lines. In this chapter, a simplified system, that is the integral control system of a pneumatic transmission line, is investigated. The control system is shown in Fig.7. In Fig.8, step responses $p$ " of the integral control system for $R_{N}=20,6$ and 2 are presented. In all cases, nondimensional integral times $T_{1}$ were 4.0 .

As shown in Fig.8(a), since signal transmission characteristics of a pneumatic line whose nondimensional parameter $R_{N}$ equaled 20 were resonant, the step responses of the closed loop system with such a pneumatic line showed considerably resonant characteristics. The step responses by use of the reduced model $G_{1}\left(s^{\prime}\right)$ showed a good agreement with the exact solution except the rising part and the first cycle of the responses. In the case of $R_{N}=6$, similar to the case of $R_{N}=20$, the reduced model's solution well agreed with the exact solution except a rising part of the responses. These differences were caused by differences between delayed time of a reduced model and that of the exact solution. Needless to say, nondimensional delayed time of a real pneumatic line $\left(T_{l}^{\prime}\right)$ equals unity. In the case of $R_{N}=2$, the step responses by use of the reduced model $G_{2}\left(s^{\circ}\right)$ strictly coincided with the exact solution. This case corresponds to the case that a pneumatic line is long and narrow. In this case, since pressure wave is considerably damped by viscosity and heat dissipation, signal transmission characteristics are found to be almost accurately expressed by a model of a first order lag element and a delayed time element. The step responses for $R_{N}=2$ overshot the steady state value.

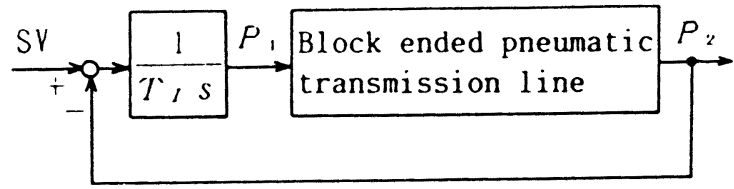

Fig7. Integral control of a pneumatic transmission line

\section{EXPERIMENTS}

Experiments were conducted using pneumatic lines usually utilized in real process control systems. In experiments, frequency responses and step responses were tested. And experimental results were compared with reduced model's results. In Fig.9, frequency responses of a pneumatic line which was $20.2 \mathrm{~m}$ in length and $4 \mathrm{~mm}$ in diameter are presented. The nondimensional parameter $R_{N}$ of the line was 4.96. Transient pressure was measured by semiconductor pressure sensors whose head space was filled with silicone oil in order to avoid unfavorable effects of head space volume. In Fig.9, plots represent experimental results and the abscissa is nondimensional angular frequency $\omega^{\prime}$. In
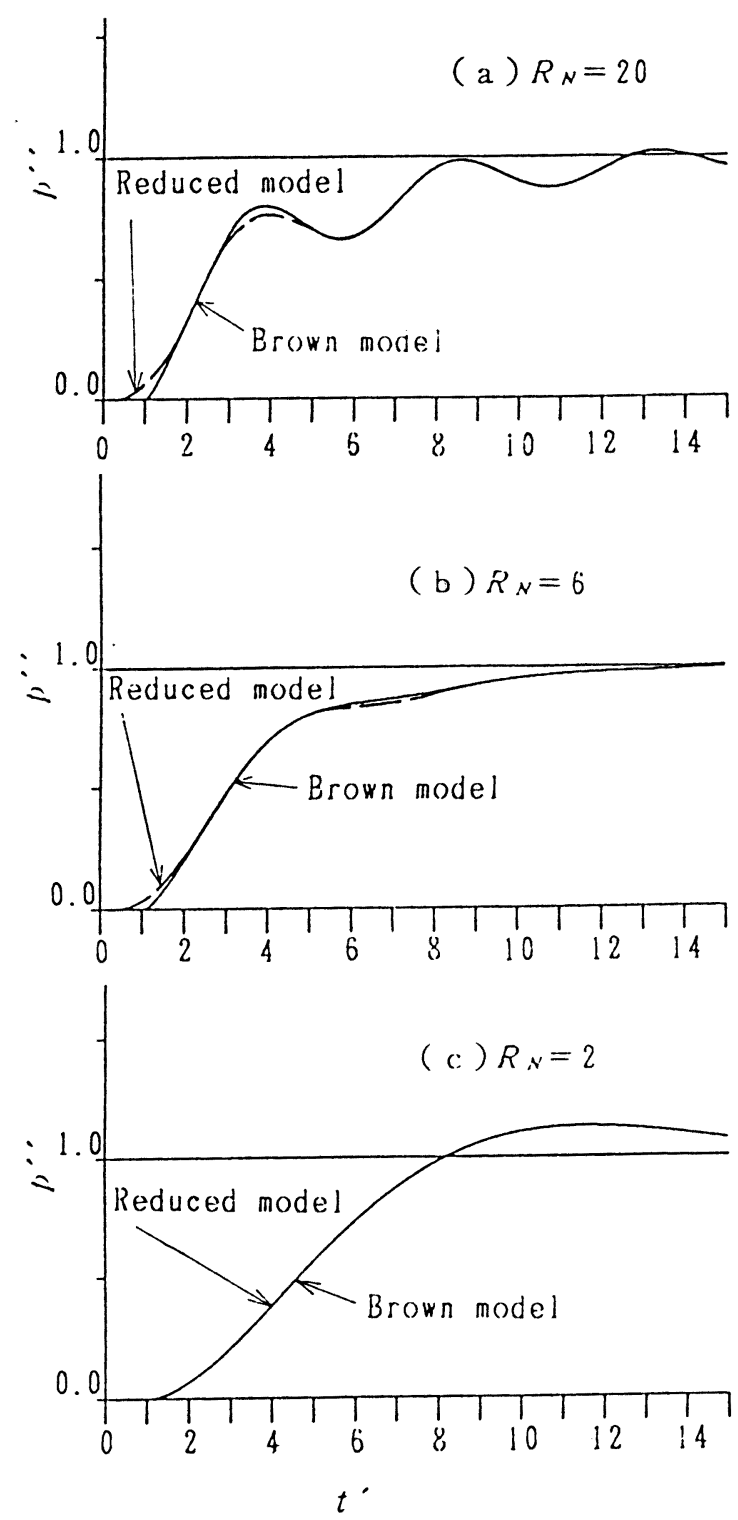

Fig. 8 Step responses of integral control loop of a transmission line 
this case, since wave traveling time was $0.0594 \mathrm{~s}$, unity of nondimensional angular frequency $\left(\omega^{\prime}=1\right)$ corresponded to $4.21 \mathrm{~Hz}$. Solid line represents the exact solution ( the Brown model ), the broken line represents the solution by the reduced model proposed in this investigation. Gain of reduced model showed a good agreement with the exact solution and experimental results up to the first resonant frequency. But at higher frequency, differences of the gain between the reduced model and the exact solution ( or experimental results ) become large. When a pneumatic transmission line is used as an element of a control system, the difference may not cause so much error in calculating dynamic responses of a total control system. Because the phase lag of a pneumatic transmission line becomes larger
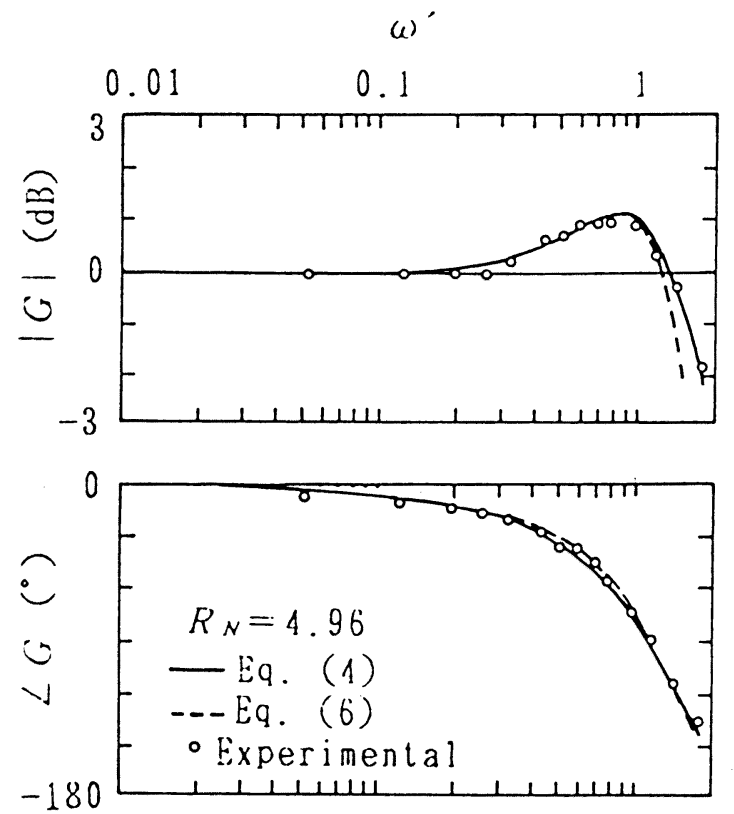

Fig.9 Experimental results of frequency responses

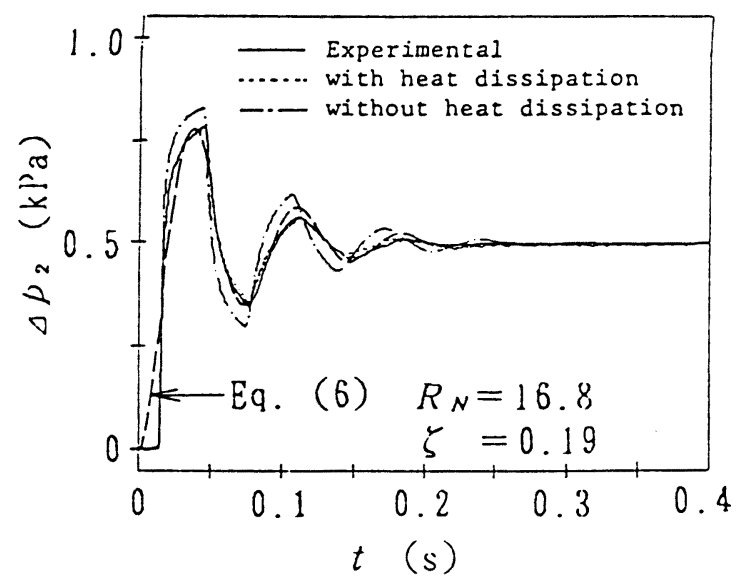

Fig. 10 Experimental results of step responses than $90^{\circ}$ at such a high frequency. On the one hand, since the reduced model has been so determined that the phase lag of the reduced model equals that of the exact solution at resonant frequency, the phase lag of reduced model showed a good agreement with the exact solution.

In Fig.10, step responses of a line which was $15.1 \mathrm{~m}$ in length and $4 \mathrm{~mm}$ in diameter are presented. Nondimensional parameter $R_{N}$ of this line was 16.8. So the line had cosiderably resonant characteristics. The solid line is experimental results, the broken line is calculated results of the reduced model and the dotted line is the exact solution (with heat dissipation). Experimental results showed a good agreement with the exact solution. Characteristics of traveling pressure wave were clearly shown. In order to investigate the wave damp caused by heat dissipation, calculated results which were performed on the assumption of adiabatic compression ( that is, without heat dissipation ) are also presented. Calculated results by the reduced model presented by the broken line can not represent details of transient responses, but the outline of transient responses can be obtained.

\section{CONCLUSION}

The reduced model which can represent signal transmission characteristics of a block ended pneumatic transmission line has been proposed. And the reduced model is compared with the exact solution and experimental results. Dynamic characteristics of a line are clearly classified as resonant region and nonresonant region according to nondimensional parameter $R_{N}$ which represents pressure wave transmission characteristics. Reduced models for each region can be determined by only two or three parameters. Values of these parameters are able to be quite easily obtained from graphs (Fig.4 or Fig.5). The reduced model proposed in this paper needs only a few parameters. Nevertheless, the model can represent signal transmission characteristics of a pneumatic line. Therefore the new reduced model is very useful for designing a real control systen containing pneumatic lines.

\section{REFERENCES}

(1) Y.SAKARAGI, Y.YONEZAKA and M.MIYAKE, Survay of Studies on the Dynamics of Pneumatic Transmission Lines, Trans.Jpn.Soc.Instrum. \& Control Eng., 
(in Japanese), Vol.2, No.1, (1966), 63.

(2) M.YOSHIOKA and Y.MORIKAWA, A Method of Transient Response Calculation for the Fluid Circuit Systems on the Basis of Frequency Characteristics, Trans.Jpn. Soc.Instrum. \& Control Eng., (in Japanese), Vol.15, No.1, (1979), 135.

(3) C.B.Schuder, and R.C.Binder, The Response of Transmission Lines to Step Inputs, Trans. ASME, J.Basic Eng., Vol.18, (1959), 578.

(4) F.T.Brown, The Transient, Response of Fluid Lines, Trans. ASME, Series D, Vol.84, (1962), 547.

(5) T.NISHIHARA, T.SAWAMURA and T.WATANABE, The Frequency Characteristics of Pneumatic Transmission Lines used in Automatic Control Systems, Trans.Jpn. Soc.Instrum. \& Control Eng., (in Japanese), Vol.25, No.115, (1956), 230.

(6) M.de Bacci, Propagation in Continuous Media, Process Control and Automation, Vol.10, No.4, (1963), 143.

(7) B.W.Anderson, The Analysis and Design of Pneunatic Systems, John Viley \& Sons, (1967).

(8) R.L.Woods, A First-order Square-root Approximation for Fluid Transmission Line, Fluid Transmission Dynamics ASME, (1983), 37.

(9) C.Y-Y.Hsue and D.A.Hullendr, Modal Approximation for the fluid Dynamics of Hydro and Pneumatic Transmission Line, Fluid Transmission Dynamics ASME (1983), 51.

(10) A.KITAGAWA, T.KAGAWA and T.TAKENAKA, High Speed and Accurate Computing Method for Transient Response of Pneumatic Transmission Line Using Characteristics Method, Trans.Jpn. Soc.Instrum. \& Control Eng., (in Japanese), Vol.20, No.7, (1984), 648. 